\title{
A Platform for Quantifying Atrial Structural Remodelling
}

\author{
Orod Razeghi ${ }^{1}$, Rashed Karim ${ }^{1}$, John Whitaker ${ }^{1}$, Catalina Tobon-Gomez ${ }^{1}$, Steven Niederer ${ }^{1}$ \\ ${ }^{1}$ Kings College London, London, UK
}

\begin{abstract}
Cross sectional imaging tools are increasingly used prior to cardiac electrophysiology procedures. Cardiac Magnetic Resonance (CMR) imaging provides detailed anatomic information that can quantify structural remodelling of the atrial chambers. Atrial Late Gadolinium Enhanced (LGE) CMR may be used to identify fibrotic change in the atrium.

A standardised and transparent approach to the analysis of CMR imaging would facilitate a broader applicability of such tools for complex image analysis. Furthermore, an assessment of the utility of such tools beyond highly specialised centres would be possible.

In this paper, we introduce an open source platform, provide standardised solutions for extracting information from clinical images, and propose standard definitions of the atrial anatomy for comparing clinical indices.
\end{abstract}

\section{Introduction}

Contemporary imaging techniques form an important part of a comprehensive assessment of patients with cardiovascular disease. They have the potential to provide highly detailed structural and functional information that may be useful for patient selection and procedural planning. Cross sectional imaging tools are increasingly used prior to elective cardiac electrophysiology procedures.

Cardiac Magnetic Resonance (CMR) imaging is established as the optimal modality for myocardial tissue characterisation. Ventricular imaging is the gold standard noninvasive technique for the identification, quantification and localisation of scar. Atrial Late Gadolinium Enhanced (LGE) CMR may be used to identify fibrotic change in the atrium [1], a marker of adverse structural remodelling with implications for procedural success. Cardiac Computed Tomography (CCT) provides higher resolution structural information and has been used to identify changes in atrial myocardial thickness [2].

When considering fibrosis and global changes in tissue thickness, it is of critical importance to restrict the assessment to the body of the atrium, and exclude the Pulmonary Veins (PV), which have different tissue dimensions and characteristics. Inclusion of PV tissue in averaged calculations has the potential to introduce significant errors in global fibrosis scores. Furthermore, atrial appendage has highly varied anatomy and size, which may bias the analysis. Registration between cross sectional imaging and Electro-Anatomic Mapping studies is frequently helpful and an accurate registration between modalities would be facilitated by a standardised geometric definition of the PV ostia.

We propose standardised methods for analysing clinical images and standard definitions of the atrial anatomy to facilitate the registration between clinical modalities.

\section{Background}

Several cardiac imaging modalities are capable of visualising the Left Atrium (LA) and enabling the quantification of both structural and functional properties of this cardiac chamber. LGE CMR has been extensively used to detect and quantify Atrial Fibrosis (AF). The Utah group have refined this technique and developed a staging system to quantify the degree of fibrosis [3].

Similar findings to the Delayed-Enhancement MRI Determinant of Successful Radio frequency Catheter Ablation of Atrial Fibrillation (DECAAF) study [1] suggest that patients with more extensive LA LGE before ablation have a higher risk of AF recurrence. However, the widespread adoption of pre-procedural LA LGE assessment has been limited by technical challenges associated with acquiring and analysing imaging outside centres with specialist expertise in this area [4].

Changes in LA myocardial wall thickness have been recognised as a crucial part of the remodelling process observed in patients with AF. Cardiac CT has high spatial resolution and true volume coverage of the heart turns it into an attractive modality for the quantification of structural abnormalities [5]. There is evidence that progressive changes in atrial wall thickness occur in patients with increasing age, AF, and other pathologies [6]. However, automated measurement techniques and standardised algorithms for the reproducible identification of the epicardial and endocardial borders of the LA using cardiac CT would refine the ability to objectively assess these changes. 
Currently, advanced CT and MRI techniques are available to characterise atrial abnormalities. Nevertheless, none of these modalities have yet become a routine part of clinical practice. While a small number of highly specialised centres have been reporting success with fibrosis and scar imaging [7], these results have not always been reproduced outside of these centres [4]. These failures may have been contributed to by limited image processing tools.

\section{Proposed Workflow}

The distribution of software developed in academia has always been a challenge. Distribution by source is an option but the requirement for the end user to deploy the compatible system architecture often results in limited implementation of open source tools. Possible solutions include the Medical Imaging Interaction Toolkit (MITK) platform developed by researchers and engineers of Mint Medical and the German Cancer Research Centre in Heidelberg. Our platform was developed as an MITK based framework extended with custom plugins for fibrosis quantification and atrial wall thickness calculation. It is available as source code and in binaries for Linux and OSX.

\subsection{Fibrosis Quantification}

\subsubsection{Image Processing}

In the first stage of the workflow for fibrosis quantification, we convert the CMR datasets from their original DICOM format into NifTI images. Besides the format conversion, we developed a cropping tool and an isotropic down sampling regime to reduce the size of each image stack to improve processing times.

Segmentation is a necessary step to extract the LA anatomy from the preoperative scans. The LA has a very thin myocardial wall making it difficult to image at even the best resolutions available. Contrast-enhanced MR Angiography (CEMRA) scans allow confident identification of the endocardial atrial surface. Consistent with the majority of LGE assessment algorithms, we rely on extracting the blood pool from CEMRA to segment the LA.

The LA is surrounded by other anatomical structures that appear with similar image intensity as the blood pool. These structures hinder the robust quantification of fibrosis. An effective truncation tool to correct the blood pool segmentations proves to be essential.

\subsubsection{Ostia Localisation and Truncation}

Due to a lack of clear anatomical landmarks, defining the boundary between the LA body and each PV is not trivial. In this study, we developed an automatic approach to obtain a standardised and consistent 3D definition of the PV ostia.

After the initial image processing steps discussed previously, an endocardial surface model is created from the binary segmentation using the marching cubes algorithm, as implemented in the Medical Image Registration ToolKit (MIRTK) libraries. Our localisation approach makes use of a Voronoi diagram extracted from the surface mesh and its corresponding centrelines [8]. As the centreline's section enters the LA body, the maximum diameter of the surrounding structure increases significantly. This point was used to identify the ostium, as illustrated in Fig. 1. The image is clipped at the ostium point and the isolated PV is used to relabel the original segmentation image.

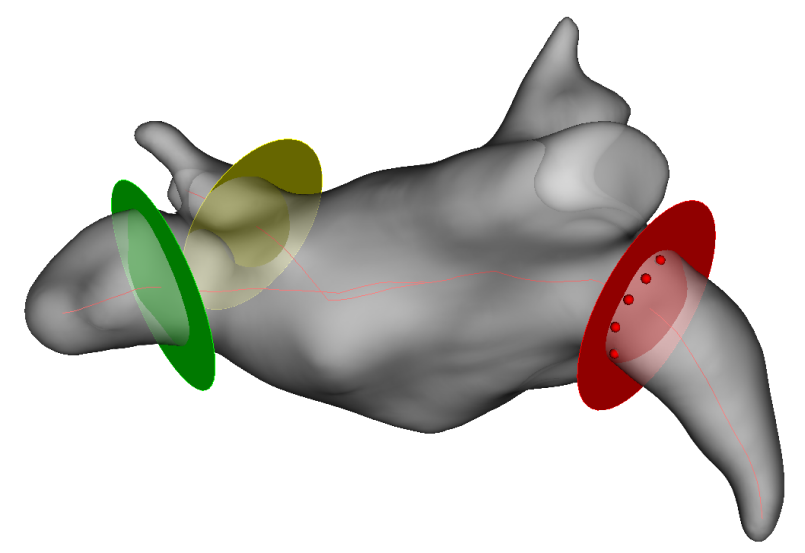

Figure 1. Ostia points identified from centrelines.

The PV arrangements and the LA Appendage (LAA) differ significantly between patients, which limit the use of cropping approaches such as that described by TobonGomez et al. [9]. In their truncation method PV clipping is performed with an infinite plane perpendicular to its corresponding centreline rendering it susceptible to additional unwanted cropping of atrial body within that plane.

We devised three types of truncation methods with different levels of manual interventions, as illustrated in the three colours in Figure. 1. The first and fully automatic clipper relies on the Vascular Modelling Toolkit (VMTK) Polydata Centreline Sections class, which computes geometric properties of sections located along centrelines. The geometric information defines a contour for the inner walls of the veins. The shape of walls originally in the Visualisation Toolkit (VTK) polydata format are converted into image stencils and used as dynamically shaped clippers to truncate the veins.

The second type of truncation method exploits the VTK implicit functions. Implicit functions are real valued functions defined in 3D space, $w=F(x, y, z)$. They require two primitive operations: the ability to evaluate the function, and the function gradient at a given point. The im- 
plicit function divides space into three regions: on the surface $F(x, y, z)=w$, outside of the surface $F(x, y, z)>c$, and inside the surface $F(x, y, z)<c$. Implicit functions are remarkably powerful, since it is possible to represent almost any type of geometry, especially if a boolean combinations of these functions are used. The intersection of an infinite plane in conjunction with a user defined sphere creates a ring shaped geometry, which results in a convex and flexible shape for the truncation of veins.

As discussed before, the structural anatomy of PVs can be very diverse. There are cases where none of the other two truncations methods described above would be suitable. Our third method is a purely manual intervention. The user picks a number of seeds on the surface mesh visualised in an interactive renderer to define a contour. These seeds generate a polydata surface, which can be stencilled and used for clipping of the veins. We have found the wide variation in patient anatomy and scan resolution make a manual tool such as this essential for those cases not amenable to automated vein clipping.

The Mitral Valve (MV) is a useful anatomic boundary between the LA and the left ventricle. In order to prevent the erroneous inclusion of ventricular tissue in assessment of AF, the implicit function and VTK mesh clipping algorithms are used to define a sphere and remove the MV from the main body of the atrial chamber.

\subsubsection{Evaluation of Fibrosis}

To project the MR signal intensities onto the 3D surface created previously from the CEMRA image, it is necessary to fuse this surface with the LGE images. The first step in the fusion is to register the masked CEMRA scan to the LGE images using a rigid transformation with 6 degrees of freedom, as implemented in MIRTK. The second step is to transform the endocardial segmentation to the new space.

Once registered, fibrosis is assessed by the signal intensity within the LGE image across a normal projected from the registered endocardial surface, as reported in [10]. The platform allows user to select the distance of the projection and the numerical output that is calculated as either a mean or maximum intensity. Vectors at a normal to each vertex on the cardiac surface are created using the VTK libraries. A mean intensity projection or a maximum intensity projection is then performed along each normal vector. The value of each intensity projection is then used to create a colour coded map on the endocardial surface mesh, as illustrated in figure 2 .

The platform offers flexibility for the user to quantify fibrosis in a number of different ways. One option is to set a threshold level according to the mean voxel values in a Region Of Interest (ROI) in the atrial blood pool plus $N$ standard deviations of the ROI. Alternatively, scar can be defined as the mean of the ROI multiplied by an image

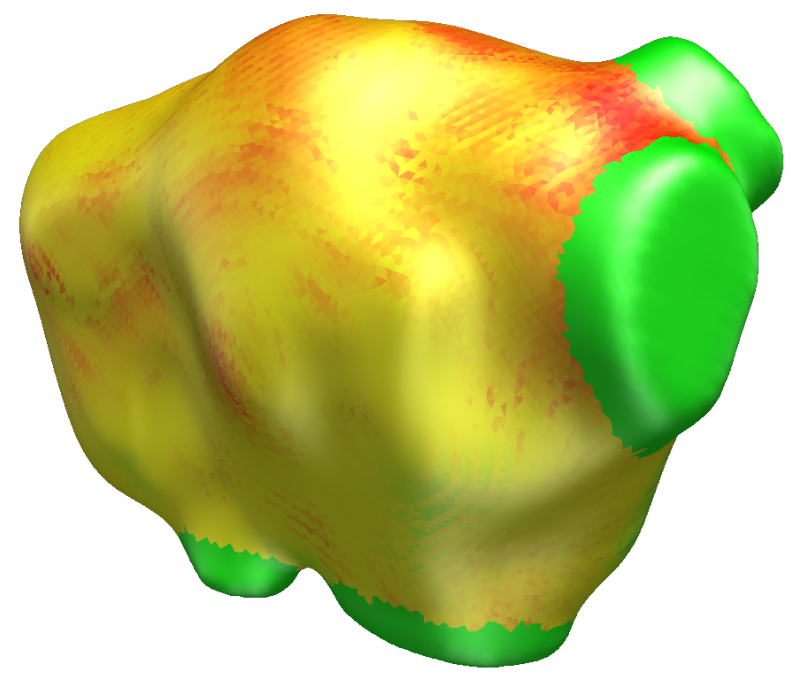

Figure 2. Max intensity projection on an atrial surface. Clipped areas are coloured in green.

intensity ratio set by the user. The total scar quantification is assessed as a percentage of the total cardiac surface.

\subsubsection{Experimental Results}

Table 1 summarises the scar quantification results in percentages. A dataset of 5 random CMR scans were initially selected to demonstrate the proof of concept and the applicability of the platform. The second and third columns in the table display the scar values when measured with image intensity ratios of 0.97 and 1.61 , respectively. The fourth column lists the scar results when measured according to the mean voxel values of the blood pool plus 3.3 times of its standard deviation. Atrial sphericities for these patients are also included in the same table. More comprehensive experiments are planned for future to fully evaluate the intra-observer and inter-observer variabilities in wider range of datasets.

Table 1. Scar Quantification Results in Percentage

\begin{tabular}{c|cccc}
\hline \hline CMR & IIR & IIR & Mean & Atrial \\
Dataset & $(0.97)$ & $(1.61)$ & $(+3.3 * S D)$ & Sphericity \\
\hline Patient 1 & 41.05 & 00.01 & 00.12 & 76.70 \\
Patient 2 & 37.76 & 00.00 & 00.00 & 75.27 \\
Patient 3 & 39.94 & 00.00 & 00.01 & 85.71 \\
Patient 4 & 96.52 & 94.61 & 94.79 & 82.22 \\
Patient 5 & 33.28 & 00.00 & 00.04 & 84.19 \\
\hline \hline
\end{tabular}

\section{Future Work}

A similar workflow was initially designed to calculate the atrial wall thickness. The same truncation algorithms 
could be used to remove PVs and the appendage from the scans. During the testing stages of the atrial wall thickness calculations, one of the issues that we encountered was the mislabelling of the endocardial and epicardial surfaces. After careful examinations, we realised that in places where the input segmentation is one voxel thin, the endocardial and epicardial surfaces meet and mesh points are consequently shared between the two surface mainly due to the Computational Geometry Algorithms Library (CGAL) [11] smoothing procedures. We initiated attempts to utilise the Tarantula meshing tool, developed by CAE Software Solutions, to remedy the aforementioned issue but further investigation is still required.

In summary to calculate the atrial wall thickness, the segmented atrial myocardium is used to produce a highresolution tetrahedral mesh using the CGAL toolbox. This mesh is processed using an in-house software, which identifies the endocardial and epicardial surfaces. Subsequently, the Laplace equation: $\nabla^{2} u=0$ is solved with Dirichlet boundary conditions assigned at the endocardial $u=0$ and epicardial $u=1$ surfaces.

The Laplace solution generates a series of smooth and non-intersecting iso-potential surfaces nested between the two boundaries. At each endocardial node, a path is defined by considering the normal projection from the endocardial surface to the first iso-potential field surface. From the projected point on the first iso-potential field surface, a second normal is projected to the subsequent iso-potential surface, a process which is repeated until the epicardial surface is reached.

A wall thickness is associated on each endocardial node as the sum of the magnitudes of the normal projections comprising a continuous path from the endocardial to the epicardial surface. This generates single, unique measurements of thickness that are independent of starting position across the atrium including the LAA.

\section{Discussion}

Whilst further experiments would be beneficial for the fibrosis quantification process and the atrial wall thickness calculations would need adjustments, the tools reported here provide a standard and open source platform for clinicians to quantify and evaluate the structural remodelling of the atrial chamber.

This platform is publicly accessible and offers the flexibility to implement and share other image processing strategies amongst academic groups. As such, it has the potential to increase the transparency when complex image processing techniques are carried out between and beyond academic centres. It further increases collaboration between groups working on similar clinically important image processing challenges.

\section{References}

[1] Marrouche N, Wilber D, Hindricks G, et al. Association of atrial tissue fibrosis identified by delayed enhancement MRI and atrial fibrillation catheter ablation: the DECAAF study. JAMA 2014;311(5):498-506.

[2] Bisbal F, Guiu E, Cabanas-Grandio P, Berruezo A, PratGonzalez S, Vidal B, Garrido C, Andreu D, FernandezArmenta J, Tolosana JM, Arbelo E, de Caralt TM, Perea RJ, Brugada J, Mont L. Cmr-guided approach to localize and ablate gaps in repeat af ablation procedure. JACC 2014; 7(7):653-663.

[3] Higuchi K, Akkaya M, Akoum N, Marrouche NF. Cardiac mri assessment of atrial fibrosis in atrial fibrillation: implications for diagnosis and therapy. Heart 2014;100(7):590 596.

[4] Sramko M, Peichl P, Wichterle D, et al. Clinical value of assessment of left atrial late gadolinium enhancement in patients undergoing ablation of atrial fibrillation. International Journal of Cardiology 2015;179:351-357.

[5] Whitaker J, Rajani R, Chubb H, Gabrawi M, Varela M, Wright M, Niederer S, O'Neill MD. The role of myocardial wall thickness in atrial arrhythmogenesis. Europace May 2016;18(12):1758-1772.

[6] Nakamura K, Funabashi N, Uehara M, et al. Left atrial wall thickness in paroxysmal atrial fibrillation by multislice-CT is initial marker of structural remodeling and predictor of transition from paroxysmal to chronic form. International Journal of Cardiology 2011;148(2):139-147.

[7] Habibi M, Lima JA, Khurram IM, et al. Association of left atrial function and left atrial enhancement in patients with atrial fibrillation: a cardiac magnetic resonance study. Circulation Cardiovascular Imaging 2015;8(2).

[8] Piccinelli M, Veneziani A, Steinman DA, Remuzzi A, Antiga L. A framework for geometric analysis of vascular structures: application to cerebral aneurysms. IEEE Transactions on Medical Imaging Aug 2009;28(8):1141-1155.

[9] Tobon-Gomez C, Geers AJ, Peters J, et al. Benchmark for algorithms segmenting the left atrium from 3D CT and MRI datasets. IEEE Transactions on Medical Imaging Jul 2015; 34(7):1460-1473.

[10] Knowles BR, Caulfield D, Cooklin M, et al. 3D visualization of acute RF ablation lesions using MRI for the simultaneous determination of the patterns of necrosis and edema. TBME Jun 2010;57(6):1467-1475.

[11] The CGAL Project. CGAL User and Reference Manual. 4.10 edition. CGAL Editorial Board, 2017.

Address for correspondence:

Cardiac Electro Mechanics Research Group

Division of Imaging Sciences and Biomedical Engineering

King's College London

St. Thomas' Hospital

London SE1 7EH 